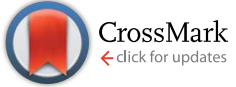

Cite this: RSC Adv., 2015, 5, 10611
Received 25th September 2014 Accepted 24th December 2014

DOI: $10.1039 / c 4 r a 11177 j$

www.rsc.org/advances

\section{One-pot synthesis of magnetic molecularly imprinted microspheres by RAFT precipitation polymerization for the fast and selective removal of $17 \beta$-estradiol $\uparrow$}

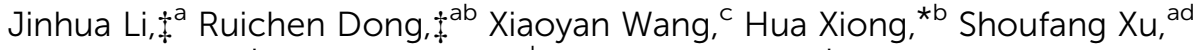 \\ Dazhong Shen, ${ }^{\star c}$ Xingliang Song ${ }^{d}$ and Lingxin Chen ${ }^{\star a}$
}

\begin{abstract}
A facile strategy was developed to prepare magnetic molecularly imprinted microspheres (MMIMs) for the selective recognition and effective removal of 17 -beta-estradiol (17 $\beta$-E2) by reversible additionfragmentation chain transfer precipitation polymerization. One-pot synthesis was employed, which could simplify the imprinting process and shorten the experimental period. The resultant MMIMs displayed fast kinetics and high binding capacity, and the adsorption processes followed LangmuirFreundlich isotherm and pseudo-second-order kinetic models. Excellent recognition selectivity toward $17 \beta$-E2 was attained over other phenolic estrogens such as 17-alpha-E2, estriol and estrone. The magnetic property of MMIMs provided fast and simple separation, and the recycling process for magnetic solid phase extraction (MSPE) was sustainable at least five times without obvious efficiency decrease. Furthermore, the MMIMs-MSPE presented satisfactory recoveries within $71.7-108.3 \%$ with the precisions of $1.1-6.0 \%$ for spiked $17 \beta$-E2 in water, soil and food samples. The developed MMIMs-based method proved to be a convenient and practical way in sample pretreatment and targeted pollutants removal.
\end{abstract}

\section{Introduction}

Estrogens, one class of important endocrine disrupting chemicals (EDCs), have become a large concern due to their widespread presence and possible adverse impacts on the endocrine systems in wildlife and humans. Among estrogens, the most potent 17-beta-estradiol (17 $\beta$-E2), a type of phenolic estrogen (PEE), can damage the endocrine systems and even cause cancer and death at low-ng $\mathrm{L}^{-1}$ concentrations. ${ }^{\mathbf{1 , 2}}$ Moreover, it is difficult to separate by conventional treatment methods in complex matrices, resulting in the formation of by-products with even higher endocrine disrupting actions., ${ }^{3,4}$ Thus, recognition,

${ }^{a}$ Key Laboratory of Coastal Environmental Processes and Ecological Remediation, Shandong Provincial Key Laboratory of Coastal Environmental Processes, Yantai Institute of Coastal Zone Research, Chinese Academy of Sciences, Yantai 264003, China. E-mail: lxchen@yic.ac.cn; Fax: +86 535 2109130; Tel: +86 5352109130

${ }^{b}$ State Key Laboratory of Food Science and Technology, Nanchang University, Nanchang 330047, China. E-mail: huaxiong100@126.com; Fax: +867916634810; Tel: +867916634810

${ }^{c}$ Key Lab in Molecular and Nanomaterials Probes of the Ministry of Education of China, College of Chemistry, Chemical Engineering and Materials Science, Shandong Normal University, Jinan 250014, China. E-mail: dzshen@sdnu.edu.cn; Fax: +8653182615258; Tel: +8653186180740

${ }^{d}$ School of Chemistry \& Chemical Engineering, Linyi University, Linyi 276005, China $\dagger$ Electric supplementary information (ESI) available. See DOI: 10.1039/c4ra11177j \$ Equally contributed to this work. determination and elimination of $17 \beta$-E2 in complicated matrices at low concentrations have great practical significance and attract increasing studies, but still face severe challenges especially for selective separation, enrichment and removal. ${ }^{5}$

Recently, a number of reports using versatile, robust and cost-effective molecularly imprinted polymers (MIPs) $^{6}$ to specifically recognize, detect and remove $17 \beta$-E2 from polluted water and foods have been demonstrated. For example, Ma et al. prepared selective core-shell MIPs of $17 \beta$-E2 on the surface of silica nanoparticles. ${ }^{7}$ Shi et al. introduced molecularly imprinted solid phase extraction (MISPE) combined with HPLC to detect trace $17 \beta$-E2 in different dairy and meat samples. ${ }^{8}$ Noir et al. fabricated macroporous MIP/cryogel composite systems for removing trace endocrine disrupting contaminants. ${ }^{9}$ Usually, MIPs are synthesized by traditional free radical polymerization, whereas the rate of chain propagation cannot be controlled, resulting in a broad size distribution. The introduction of reversible addition-fragmentation chain transfer (RAFT) polymerization techniques into the molecular imprinting strategy has attracted significant interest, which can solve the above problem..$^{\mathbf{1 0 - 1 4}}$ For examples, Pan et al. described an approach to obtain water-compatible MIPs by the facile surface-grafting of functional polymer brushes via RAFT polymerization. ${ }^{10}$ Titirici et al. prepared thin film MIPs around mesoporous silica beads by adopting covalent immobilization 
of azo initiators and RAFT-mediated living radical polymerization. ${ }^{11}$ Our group presented atrazine MIPs for preconcentration of atrazine in food matrices by using di-thioesters based RAFT coupled to precipitation polymerization. ${ }^{12,13}$

Moreover, magnetic MIPs have received wide attentions since they can be easily isolated/collected and recycled by an external magnetic field and have been applied in many fields such as separation/purification, chemo/biosensing, and drug delivery. ${ }^{15-19}$ For instance, Li et al. synthesized a core-shell magnetic imprinted polymer for the fast and selective removal of EDCs. ${ }^{18}$ Moreover, our group successfully prepared photonic/ magnetic dual-responsive MIPs for recognition of caffeine. ${ }^{19}$

Herein, inspired by these studies, we developed an improved core-shell MIPs synthesis strategy for enhanced selective recognition and removal of $17 \beta$-E2 by combining RAFT precipitation polymerization and magnetic separation. One-pot synthesis was conducted, allowing all reagents to react together under proper conditions. Moreover, the resultant MIPs layer was grafted onto the surface of magnetic iron oxide beads by a RAFT agent for improving the polymerization efficiency. The obtained magnetic molecularly imprinted microspheres (MMIMs) were easily characterized by morphologies, structures, thermostability and magnetism, as well as static and dynamic adsorptions. Finally, the MMIMs were used as SPE sorbents and successfully applied to the extraction of $17 \beta-\mathrm{E} 2$, providing a practicable way in samples pretreatment and removal of trace targeted pollutants in environment and food.

\section{Experimental}

\subsection{Materials and apparatus}

$\mathrm{FeCl}_{3} \cdot 6 \mathrm{H}_{2} \mathrm{O}$, carbon disulfide $\left(\mathrm{CS}_{2}\right)$, tetraethoxysilane (TEOS) and ammonia were purchased from Tianjin Chemical Reagents Company (Tianjin, China). 17-Beta-estradiol (17ß-E2) and acetonitrile were purchased from J\&K Technology Limited (Beijing, China). 17-Alpha-estradiol (17 $\alpha$-E2) and estriol (E3) were obtained from Dr Ehrenstorfer (Germany). Estrone (E1), diethylstilbestrol (DES), hexestrol (HS), dienestrol (DS), and divinylbenzene (DVB) were provided by Sigma-Aldrich (Shanghai, China). Bisphenol A (BPA) and phenyl magnesium bromide (PMB) were supplied by Aladdin (Shanghai, China). Tetrahydrofuran (THF), acrylamide (AA), glycol and triethylamine were purchased from Kermel (Tianjin, China). 4-(Chloromethyl)-phenyltrichlorosilane (4-CPS) was purchased from Alfa Aesar (Tianjin, China). All other reagents such as 2,2'azobisisobutyronitrile (AIBN), trichloromethane, toluene and acetone were supplied by Sinopharm Chemical Reagent Co. Ltd (Shanghai, China). Prior to use, THF was refluxed over sodium and then distilled; moreover, toluene and DVB were distilled in vacuum, and AIBN and AA were recrystallized in methanol and water, respectively. Deionized water used throughout the work was produced by a Milli-Q Ultrapure water system with the water outlet operating at $18.2 \mathrm{M} \Omega \cdot \mathrm{cm}$ (Millipore, Bedford, USA).

The morphological evaluation was performed by transmission electron microscopy (TEM, JEOL-100CX-2). Infrared spectra were recorded using Fourier transform infrared spectrometer (FT/IR-4100, Thermo Nicolet Corporation, USA).
Thermal gravimetric analysis (TGA) was carried out from room temperature to $800{ }^{\circ} \mathrm{C}$ with a heating rate of $10^{\circ} \mathrm{C} \mathrm{min}^{-1}$ under nitrogen environment by thermal gravimetric analyzer (Mettler 5 MP), presenting TGA and derivative thermogravimetry (DTG) data. Magnetic property was measured by vibrating sample magnetometer (VSM, Lake Shore 7410, Beijing, China). UV-Vis spectra were recorded using a Thermo Scientific NanoDrop 2000/2000c spectrophotometer (Thermo, USA). $\mathrm{N}_{2}$ adsorptiondesorption isotherms were examined with Beishide instruments (3H-2000PS4, Beijing) for Brunauer-Emmett-Teller (BET) analysis to determine the specific surface area and pore size. The amounts of analytes were determined by HPLC-UV (Skyray LC-310, Skyray Instrument Inc., China), under the optimized conditions: sample loaded, $20 \mu \mathrm{L}$; mobile phase, acetonitrile-water (v/v, $7: 3)$; flow rate, $1.0 \mathrm{~mL} \mathrm{~min}^{-1}$; detection wavelength, $208 \mathrm{~nm}$; column, $250 \mathrm{~mm} \times 4.6 \mathrm{~mm} \mathrm{C}_{18}$ at $20^{\circ} \mathrm{C}$.

\subsection{Synthesis of $\mathrm{Fe}_{3} \mathrm{O}_{4} @ \mathrm{SiO}_{2}$ microspheres}

Magnetic $\mathrm{Fe}_{3} \mathrm{O}_{4} @ \mathrm{SiO}_{2}$ microspheres were synthesized according to a previous report ${ }^{18}$ with slight modification. $\mathrm{Fe}_{3} \mathrm{O}_{4}$ nanoparticles were firstly prepared by the solvothermal reduction method. Typically, $1.35 \mathrm{~g} \mathrm{FeCl} \cdot 6 \mathrm{H}_{2} \mathrm{O}$ was dissolved in $40 \mathrm{~mL}$ glycol in a $100 \mathrm{~mL}$ flask with vigorously stirring. Then, 2.0 $\mathrm{g} \mathrm{CH}_{3} \mathrm{COONa}$ and $3.0 \mathrm{~g}$ polyethylene glycol were added into the solution. After ultrasonic treatment for $30 \mathrm{~min}$, the solutions were transferred to a $50 \mathrm{~mL}$ Teflon-lined autoclave and reacted for $10 \mathrm{~h}$ in a $200{ }^{\circ} \mathrm{C}$ oven, and the black $\mathrm{Fe}_{3} \mathrm{O}_{4}$ resultants were collected. Subsequently, $100 \mathrm{mg} \mathrm{Fe}_{3} \mathrm{O}_{4}$ nanoparticles were dispersed into $100 \mathrm{~mL}$ ethanol-water solvent $(\mathrm{v} / \mathrm{v}, 3: 1)$ in a $250 \mathrm{~mL}$ round flask and ultrasonically treated for $10 \mathrm{~min}$. Then,

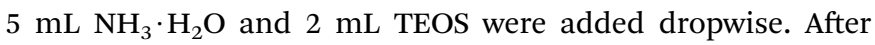
reaction for $12 \mathrm{~h}$ with constant stirring at room temperature, the brown black resultants $\mathrm{Fe}_{3} \mathrm{O}_{4} @ \mathrm{SiO}_{2}$ was washed with a lot of ethanol and water, and dried under vacuum at $50{ }^{\circ} \mathrm{C}$.

\subsection{Synthesis of $\mathrm{Fe}_{3} \mathrm{O}_{4} @ \mathrm{SiO}_{2}$-RAFT microspheres}

Magnetic $\mathrm{Fe}_{3} \mathrm{O}_{4} @ \mathrm{SiO}_{2}$-RAFT microspheres were synthesized according to the reported procedure ${ }^{20}$ with necessary modification. Briefly, $1.0 \mathrm{~g} \mathrm{Fe}_{3} \mathrm{O}_{4} @ \mathrm{SiO}_{2}$ microspheres were dispersed in $50 \mathrm{~mL}$ anhydrous toluene in a $100 \mathrm{~mL}$ three-necked flask by sonication for $15 \mathrm{~min}$, followed by the addition of $2 \mathrm{~mL} 4$-CPS and $1 \mathrm{~mL}$ triethylamine dropwise. After heating at reflux for $24 \mathrm{~h}$ under nitrogen protection, the modified $\mathrm{Fe}_{3} \mathrm{O}_{4} @ \mathrm{SiO}_{2}$ particles were washed, dried and collected. $3.0 \mathrm{~mL} \mathrm{CS}_{2}$ was added into $100 \mathrm{~mL}$ THF solution containing $10 \mathrm{~mL} \mathrm{PMB}$, and reacted for $2 \mathrm{~h}$ at $45^{\circ} \mathrm{C}$. Subsequently, $0.5 \mathrm{~g}$ modified $\mathrm{Fe}_{3} \mathrm{O}_{4} @ \mathrm{SiO}_{2}$ particles were dispersed in the mixture and stirred for $48 \mathrm{~h}$ at $65^{\circ} \mathrm{C}$ under a $\mathrm{N}_{2}$ atmosphere, and the resultant products were washed with acetone and toluene repeatedly and dried under vacuum at $60^{\circ} \mathrm{C}$ for $24 \mathrm{~h}$. The brown resultants were marked as $\mathrm{Fe}_{3} \mathrm{O}_{4} @ \mathrm{SiO}_{2}$-RAFT.

\subsection{Preparation of magnetic core-shell polymer microspheres}

Core-shell imprinted polymer microspheres for $17 \beta-\mathrm{E} 2$ were prepared by one-pot RAFT precipitation polymerization. 


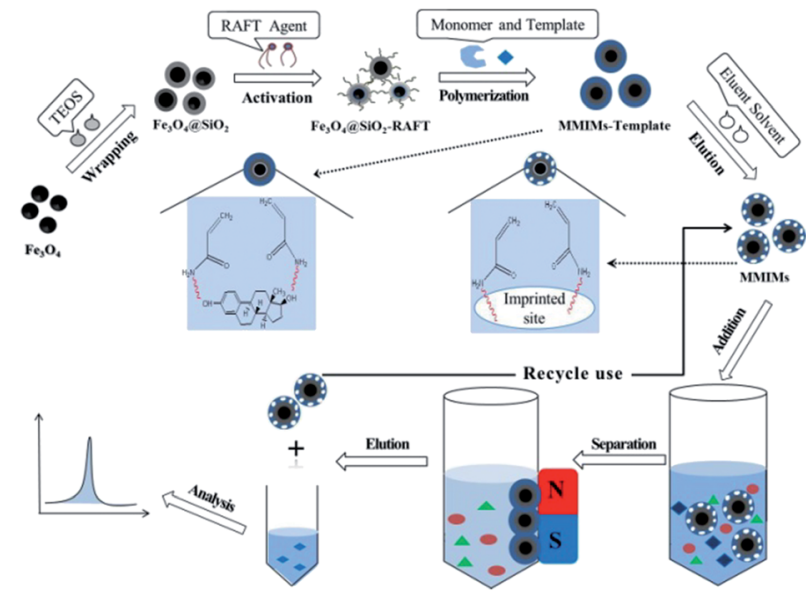

Fig. 1 Schematic illustration of the preparation process of MMIMs by RAFT precipitation polymerization, and MSPE application for recognition and removal of $17 \beta-E 2$.

Typically, $0.1 \mathrm{mmol} 17 \beta$-E2 and $0.6 \mathrm{mmol}$ AA were dispersed in $10 \mathrm{~mL}$ toluene and $40 \mathrm{~mL}$ acetonitrile solution, and then $100 \mathrm{mg}$ $\mathrm{Fe}_{3} \mathrm{O}_{4} @ \mathrm{SiO}_{2}$-RAFT, $2 \mathrm{mmol}$ DVB and $20 \mathrm{mg}$ AIBN were added. The resultant mixture was stirred at $70{ }^{\circ} \mathrm{C}$ for $24 \mathrm{~h}$ under $\mathrm{N}_{2}$ protection. The product was washed with acetone several times and then eluted by Soxhlet extraction with methanol-acetic solvent $(9: 1, \mathrm{v} / \mathrm{v})$ for $24 \mathrm{~h}$ until no template detected. The obtained gray particles, i.e. magnetic molecularly imprinted microspheres (MMIMs), were dried to constant weight under vacuum at $40{ }^{\circ} \mathrm{C}$, for use. The preparation process was schematically depicted in Fig. 1. On the other hand, magnetic nonimprinted microspheres (MNIMs) were also prepared by the same protocol without the addition of template. Moreover, as a control, $\mathrm{Fe}_{3} \mathrm{O}_{4} @ \mathrm{SiO}_{2}$ microspheres were added for the one-pot precipitation polymerization reaction directly without adding RAFT agent, and the resultant dark gray particles were named as MMIMs-Control.

\subsection{Binding property studies of the magnetic microspheres}

Binding properties of the prepared MMIMs, MNIMs and MMIMs-Control were studied in acetonitrile, which included static adsorption, dynamic adsorption and selectivity experiments. Typically, static adsorption experiments were carried out by dispersing $20 \mathrm{mg}$ polymer microspheres into $2.0 \mathrm{~mL}$ acetonitrile solutions containing different concentrations of $17 \beta-\mathrm{E} 2$ $\left(1,10,20,40,60,80\right.$ and $\left.100 \mathrm{mg} \mathrm{L}^{-1}\right)$ in $10 \mathrm{~mL}$ colorimetric tubes. After being shaken for $24 \mathrm{~h}$ at room temperature, the samples were isolated using an external magnet. The residual concentration of $17 \beta-\mathrm{E} 2$ in the supernatant solution was determined by HPLC-UV. The adsorbed amount of $17 \beta$-E2 was calculated by subtracting the residual amount from its total amount. Dynamic adsorption experiments were performed with $20 \mathrm{mg}$ polymer microspheres dispersed into $2.0 \mathrm{~mL}$ acetonitrile solutions containing $60 \mathrm{mg} \mathrm{L}^{-1} 17 \beta$-E2. After stirring for 10, 30, $60,90,120,180$ and $270 \mathrm{~min}$, the solution was separated and quantified by HPLC-UV. Selectivity experiments were conducted by comparing the binding capacities of the polymer microspheres between $17 \beta$-E2 and its structural analogues (E3, $17 \alpha$-E2, E1, BPA, DES, HS and DS). Briefly, $20 \mathrm{mg}$ polymer microspheres were dispersed into $2.0 \mathrm{~mL}$ acetonitrile solutions containing $60 \mathrm{mg} \mathrm{L}^{-1}$ of different kinds of PEEs, and after being shaken for $24 \mathrm{~h}$ at room temperature, the supernatant was quantified by HPLC-UV. All tests were determined in triplicate.

\subsection{MMIMs applied in magnetic solid phase extraction for analysis of real samples}

Seawater samples were randomly collected from the surface seawater of Yellow Sea, lake water samples were collected from Sanyuan Lake, and soil samples were obtained from a suburb, which were all located in the coastal zone of Yantai City. Yogurt samples were purchased from a local market of Laishan District. These samples were simply extracted as follows. For the seawater, lake water and yogurt samples, $1 \mathrm{~mL}$ of each sample was added into $10 \mathrm{~mL}$ acetonitrile, and after being shaken for $30 \mathrm{~min}$, the solutions were centrifuged and filtered, and the extract solutions were obtained. For the solid samples, $2.0 \mathrm{~g}$ soil samples were dispersed into $20 \mathrm{~mL}$ acetonitrile solution and then shaken for $1 \mathrm{~h}$. The supernatants were centrifuged and filtered with a $0.45 \mu \mathrm{m}$ microfiltration membrane. All the sample extract solutions were stored at $4{ }^{\circ} \mathrm{C}$ for later use.

The process of magnetic solid phase extraction (MSPE) was carried out according to a previously reported method ${ }^{21-23}$ with suitable modifications. Specifically, MMIMs of $80 \mathrm{mg}$ were dispersed into $2 \mathrm{~mL}$ sample extract solutions spiked with $17 \beta$-E2 at three concentrations $\left(0.1,1.0\right.$ and $\left.10 \mathrm{mg} \mathrm{L}^{-1}\right)$. After being shaken for $3 \mathrm{~h}$ at room temperature, the MMIMs sorbent loaded with $17 \beta$-E2 was separated from the suspension using a magnet, and the supernatant was measured by HPLC-UV. Subsequently, the $17 \beta$-E2 adsorbed onto the MMIMs sorbent was eluted with $2 \mathrm{~mL}$ of methanol-acetic acid solution $(9: 1$, $\mathrm{v} / \mathrm{v}$ ). Then, the eluent was dried and re-dissolved in $2 \mathrm{~mL}$ acetonitrile and then determined by HPLC-UV. Finally, after the extraction process, in order to reuse the same sorbent for new extraction of the $17 \beta$-E2, the MMIMs were washed with methanol-acetic acid solution and acetonitrile several times, and then the sorbent was dried at $60{ }^{\circ} \mathrm{C}$ for new extraction. The MSPE procedure is illustrated in Fig. 1.

\section{Results and discussion}

\subsection{Preparation and characterization of the MMIMs}

Fig. 1 shows the preparation process of MMIMs by a one-pot RAFT precipitation polymerization, and the MSPE procedure for MMIMs application. The core-shell MMIMs were prepared in a mild condition with a one-pot approach, which allowed all reagents to be added and to react together. The RAFT polymerization procedure was simple and avoided complex surface modification; moreover, the one-pot setting simplified the experimental process and shortened the synthetic period. Then, the obtained MMIMs were fully characterized by TEM, FT-IR, TGA, VSM and BET as follows.

Fig. 2 shows the morphologies of the products at different synthetic stages. $\mathrm{Fe}_{3} \mathrm{O}_{4} @ \mathrm{SiO}_{2}$ (Fig. 2A) and MMIMs (Fig. 2B) 


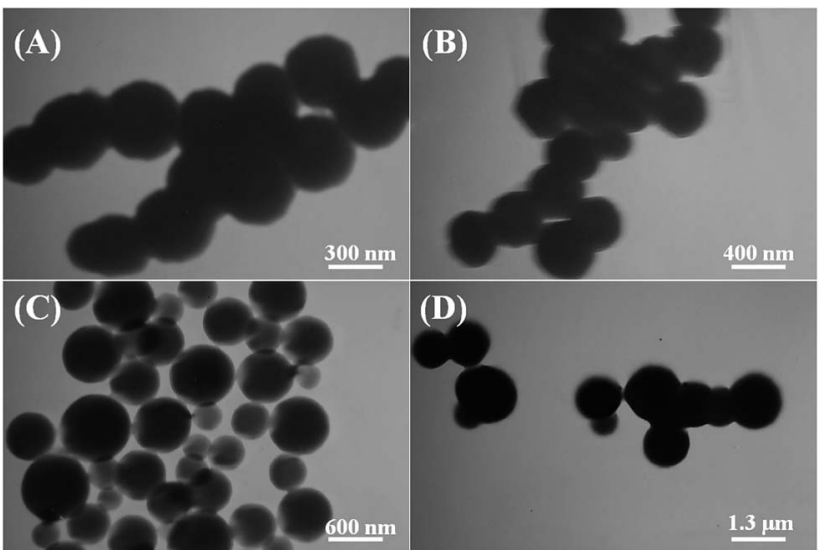

Fig. 2 TEM images of $\mathrm{Fe}_{3} \mathrm{O}_{4}\left(\mathrm{CSiO}_{2}\right.$ (A), MMIMs (B), MMIMs-Control (C) and MNIMs (D) particles.

particles were monodisperse and exhibited a regular sphere morphology, which indicated the polymerization smoothly proceeded on the surface of $\mathrm{Fe}_{3} \mathrm{O}_{4} @ \mathrm{SiO}_{2}$ by RAFT. By comparing Fig. $2 \mathrm{~A}$ and $\mathrm{B}$, the thickness of the imprinting shell layer could be attained within 10-30 nm (Fig. 2B). Owing to the thin imprinting layers, almost all the template molecules could be completely eluted and thereby produce the largest amounts of imprinted cavity sites, resulting in high binding capacities and fast mass transfer. However, the MMIMs-Control and MNIMs particles were not uniform in size distribution, with the average diameter ranging from 225 to $505 \mathrm{~nm}$ for MMIMsControl (Fig. 2C) and from 730 to $1500 \mathrm{~nm}$ for MNIMs (Fig. 2D), respectively. And the non-uniform size distribution was adverse to mass transfer. When $\mathrm{Fe}_{3} \mathrm{O}_{4} @ \mathrm{SiO}_{2}$ without further RAFT functionalization was used, imprinting layers could not form around the magnetic particles, but non-magnetic secondary particles formed by precipitation polymerization with much smaller diameters $(100-200 \mathrm{~nm})$, as shown in Fig. 2C. The secondary particles might well adhere with the magnetic particles, and thereby cause inhomogeneous size and distribution of the MMIMs-Control (Fig. 2C). For MNIMs, as seen in Fig. 2D, their sizes were quite large with a wide distribution, which was quite different from MMIMs. This was very likely owing to the absence of template in the RAFT polymerization. For the MNIMs, without the presence of template molecules, the crosslinking substances, all of which contain double bonds, could more easily form shell-layers on the surface of $\mathrm{Fe}_{3} \mathrm{O}_{4}$ @$\mathrm{SiO}_{2}$, and thereby result in thicker shell-layers and easier aggregation, presenting non-homogeneous and much larger material (Fig. 2D).

Fig. 3A shows the FT-IR spectra of $\mathrm{Fe}_{3} \mathrm{O}_{4}, \mathrm{Fe}_{3} \mathrm{O}_{4} @ \mathrm{SiO}_{2}$, $\mathrm{Fe}_{3} \mathrm{O}_{4} @ \mathrm{SiO}_{2}$-RAFT, MMIMs and MNIMs particles. The polymerization process could be evaluated according to the characteristic functional groups. The characteristic absorption band of $\mathrm{Fe}_{3} \mathrm{O}_{4}$ was at $580 \mathrm{~cm}^{-1}$ (curve a). The absorption at 803 and $1089 \mathrm{~cm}^{-1}$ could be attributed to the stretching vibration of $\mathrm{Si}-\mathrm{O}$ and $\mathrm{Si}-\mathrm{O}-\mathrm{Si}$, respectively, proving that $\mathrm{SiO}_{2}$ was successfully covered on the $\mathrm{Fe}_{3} \mathrm{O}_{4}$ particles (curve b). As seen from curve $\mathrm{c}$, the peak at $1629 \mathrm{~cm}^{-1}$ represented the stretching vibration of $\mathrm{C}=\mathrm{C}$ bond of alkene, due to the RAFT active modification on
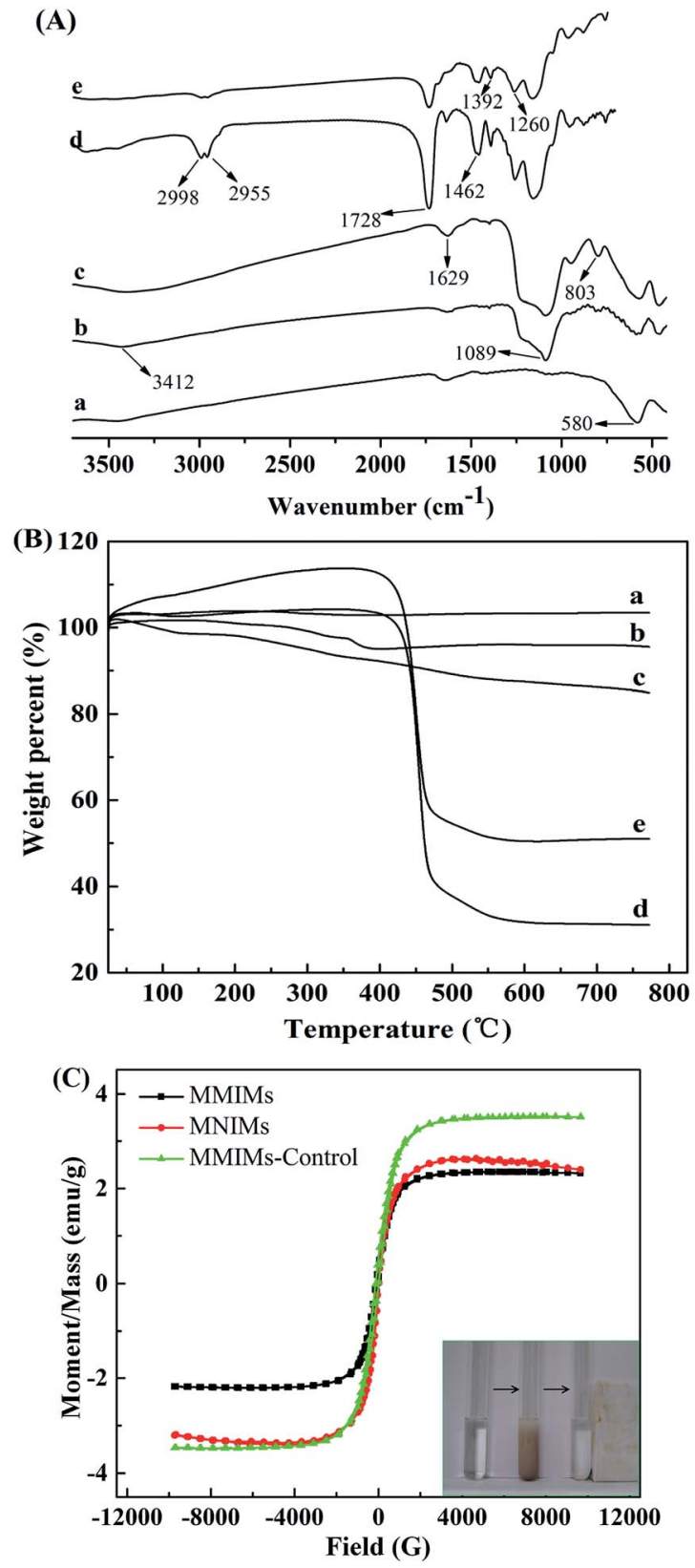

Fig. 3 (A) FT-IR spectra and (B) TGA curves of $\mathrm{Fe}_{3} \mathrm{O}_{4}$ (a), $\mathrm{Fe}_{3} \mathrm{O}_{4}\left(\mathrm{aSiO}_{2}\right.$ (b), $\mathrm{Fe}_{3} \mathrm{O}_{4} @ \mathrm{OSiO}_{2}-\mathrm{RAFT}$ (c), MMIMs (d) and MNIMs (e). (C) Magnetic hysteresis loops of MMIMs, MNIMs and MMIMs-Control, and the inset shows the dispersion and separation process of a $17 \beta-E 2$ solution in absence (left) and presence (middle) of MMIMs and in the presence of an external magnetic field (right).

the surface of $\mathrm{Fe}_{3} \mathrm{O}_{4} @ \mathrm{SiO}_{2}$. The unique absorption peak at $1728 \mathrm{~cm}^{-1}$ belonged to the stretching vibration of the $\mathrm{C}=\mathrm{O}$ bond, the typical peak at 2998 and $2955 \mathrm{~cm}^{-1}$ could be ascribed to the saturated $\mathrm{C}-\mathrm{H}$ bond, as well as the peaks at 1392 and 1260 $\mathrm{cm}^{-1}$ could be assigned to the $\mathrm{C}-\mathrm{N}$ bond, indicating the imprinted polymer layer was grafted onto the surface of $\mathrm{Fe}_{3}$ $\mathrm{O}_{4} @ \mathrm{SiO}_{2}$ successfully, as demonstrated in curve d. In addition, the peak at $1462 \mathrm{~cm}^{-1}$ belonged to the aromatic carbon-carbon bond of DVB, which was used for the preparation of the polymer. The FT-IR spectrum of MNIMs (curve e) looks exactly the 
same as that of MMIMs (curve d), except for the lower intensity. All the results of FT-IR confirmed that the core-shell structured MMIMs were successfully prepared by RAFT precipitation polymerization.

The corresponding TGA results of the above five particles were displayed in Fig. 3B. As can be seen in the figure, the TGA curve of $\mathrm{Fe}_{3} \mathrm{O}_{4}$ was almost a straight line, indicating the particles were very pure and without any impurities (curve a). There was a slight weight loss at $250-350{ }^{\circ} \mathrm{C}$ for $\mathrm{Fe}_{3} \mathrm{O}_{4} @ \mathrm{SiO}_{2}$ particles (curve b), which could be attributed to the dehydration of the $\mathrm{SiO}_{2}$ layer. As can be observed from curve c, $\mathrm{Fe}_{3} \mathrm{O}_{4} @ \mathrm{SiO}_{2}$-RAFT exhibited bigger weight loss, which may be due to the pyrolysis of RAFT active groups. The TGA curves of MMIMs (curve d) and MNIMs (curve e) particles dropped rapidly at around $450{ }^{\circ} \mathrm{C}$, suggesting that the whole imprinted and non-imprinted polymer layers on the surface of $\mathrm{Fe}_{3} \mathrm{O}_{4} @ \mathrm{SiO}_{2}$ largely decomposed under high temperature. In addition, the peak temperatures of MMIMs and MNIMs were 454.36 and $451.72{ }^{\circ} \mathrm{C}$, respectively, and the residual amounts were $25.61 \%$ and $36.30 \%$, respectively, as can be seen from the TGA results in Table S1† and DTG curves in Fig. S1. $\uparrow$ These results demonstrated that the MMIMs possessed higher polymerization efficiency and good thermal stability below $400{ }^{\circ} \mathrm{C}$.

Fig. 3C shows the magnetic hysteresis loops analysis of the MMIMs, MMIM-Control and MNIMs, and the inset illustrates the dispersion and agglomeration processes of the MMIMs. It is seen that there is a similar general shape to the three curves, although the saturation magnetization value of MMIMs is low (Table S2 $\dagger$ ). The results suggested that the prepared MMIMs were magnetically responsive. As can be seen from Table S2, $\dagger$ the MMIMs had better magnetic induction intensity, which may be caused by the strong magnetism of independent $\mathrm{Fe}_{3} \mathrm{O}_{4} @ \mathrm{SiO}_{2}$ particles that did not participate in the reaction. Consequently, the homogeneously dispersed MMIMs could go straight towards the magnet and adhere to the inner side wall of the vials when the external magnetic field was applied, and the turbid solution became clear and transparent, as evidenced in the inset of Fig. 3C, showing a fast and simple magnetic separation.

The $\mathrm{N}_{2}$ adsorption-desorption isotherms and pore diameter distribution of MMIMs, MMIMs-Control and MNIMs are shown in Fig. 4. The type IV isotherm curves with a loop were observed for MMIMs (Fig. 4A), which indicated that MMIMs had a welldefined porous structure. As can be seen in the figure, the MMIMs showed a hysteresis loop, where the desorption curve was closer but slightly leveled above the adsorption curve, representing that the MMIMs were stable with low swelling and low solvent uptake. ${ }^{24}$ The narrow pore size distribution and low average pore diameter that can be observed from the adsorption plot (Fig. 4B) suggested that the size of the cavities formed in the MMIMs matrix played an important role in the binding capacity. The structure parameters of the three microspheres obtained by BET analysis are listed in Table S3. $\dagger$ As can be seen in the figure, the specific surface area, cumulative pore volume and average pore diameter of MMIMs particles were $444.86 \mathrm{~m}^{2} \mathrm{~g}^{-1}, 0.10 \mathrm{~mL} \mathrm{~g}^{-1}$ and $4.18 \mathrm{~nm}$, respectively, while those of MMIMs-Control particles were $6.89 \mathrm{~m}^{2} \mathrm{~g}^{-1}$,
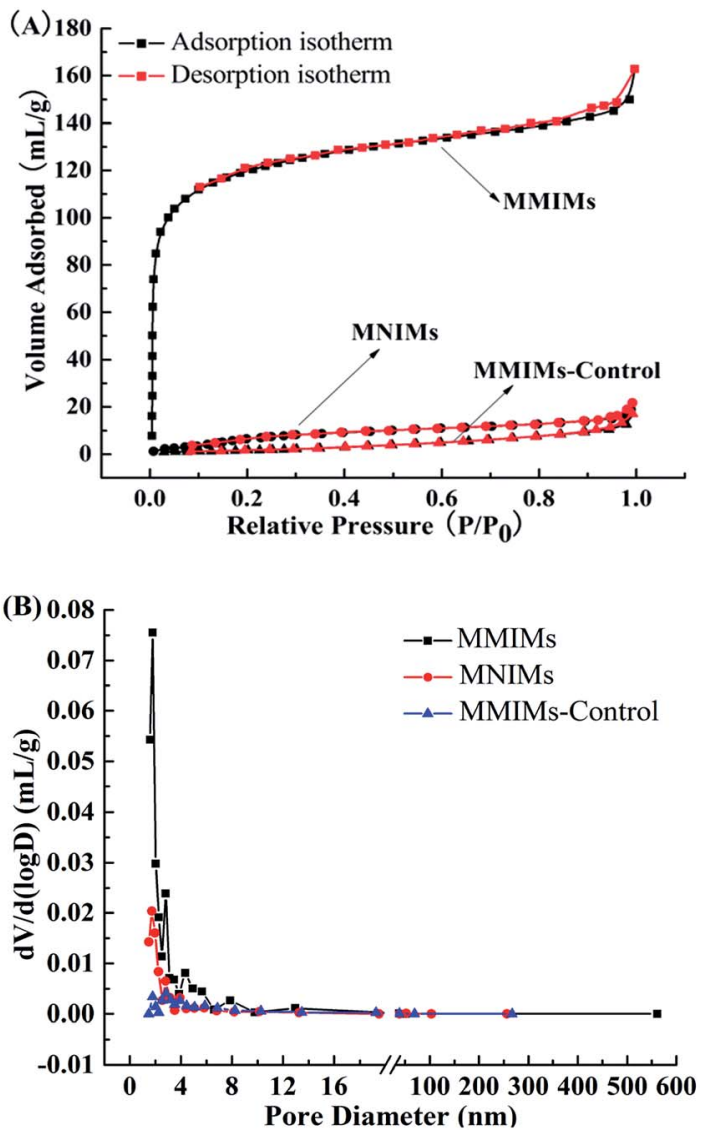

Fig. $4 \quad N_{2}$ adsorption-desorption isotherms (A) and pore diameter distribution curves (B) of MMIMs, MNIMs and MMIMs-Control.

$0.030 \mathrm{~mL} \mathrm{~g}^{-1}$ and $7.96 \mathrm{~nm}$, and those of MNIMs particles were $46.18 \mathrm{~m}^{2} \mathrm{~g}^{-1}, 0.038 \mathrm{~mL} \mathrm{~g}^{-1}$ and $4.35 \mathrm{~nm}$, respectively. Obviously, the specific surface area and cumulative pore volume of MMIMs were much larger, while the average pore diameter was slightly smaller than that of the latter two. The large specific surface area proved that MMIMs had a uniform, regular spherical structure (Fig. 2B). Generally, MIPs have slight differences in surface area and pore volume from NIPs. Here, the significantly high values of MMIMs might be easily attributed to the template functioning somehow with the RAFT polymerization. During MMIMs preparation, when the template molecules were added, the synthesis would become a doping synthesis to some extent. This would produce thinner imprinting shell-layers with smaller diameters, along with amounts of imprinted cavities, and therefore would lead to large surface area and pore volume.

\subsection{Binding properties of the MMIMs}

The binding ability of MMIMs was investigated by performing the static, dynamic and selectivity studies. Fig. 5A shows the static binding isotherms of $17 \beta$-E2 onto three polymers. As can be seen in the figure, the adsorption capacity for $17 \beta$-E2 increased quickly as its initial concentration increased. When the equilibrium concentration was higher than $60 \mathrm{mg} \mathrm{L}^{-1}$, the adsorption amounts of MMIMs became stable and its 


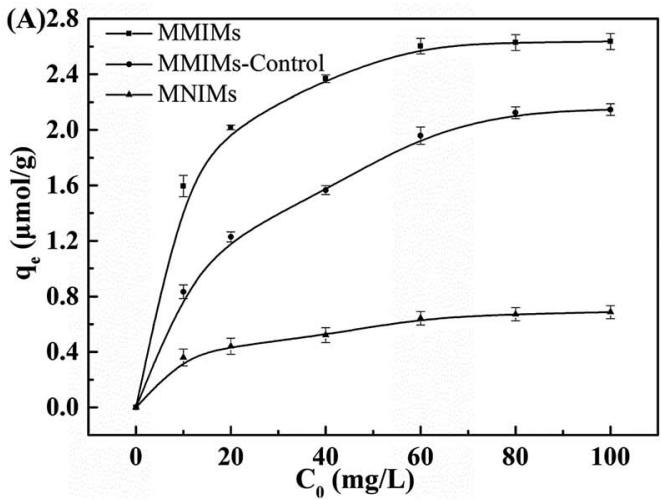

(B)
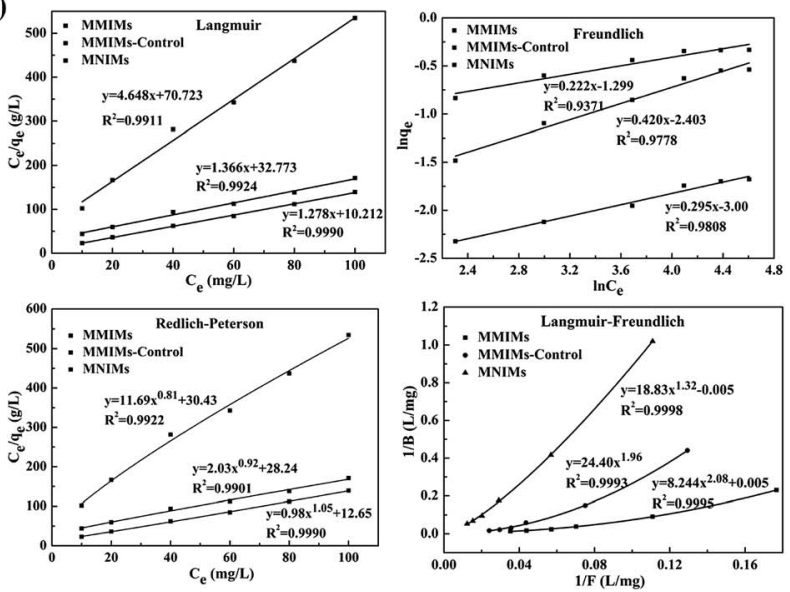

Fig. 5 (A) Static adsorption isotherms of $17 \beta-E 2$ onto MMIMs, MMIMsControl and MNIMs and (B) Langmuir, Freundlich, Redlich-Peterson and Langmuir-Freundlich isotherm models for $17 \beta-E 2$ adsorption process. Experimental conditions: V, $2 \mathrm{~mL}$; polymer, $20 \mathrm{mg}$; adsorption time, $12 \mathrm{~h}$; room temperature.

recognition sites were almost occupied by 17 $\beta$-E2. MMIMsControl and MNIMs displayed the same trends, but lower saturated adsorption amounts; hence, the MMIMs exhibited significantly high 17 $\beta$-E2 loading. Moreover, according to the Scatchard equation, ${ }^{25}$ the $K_{\mathrm{d}}$ (equilibrium dissociation constant) and $Q_{\max }$ (maximum adsorption capacity) were calculated, i.e., $K_{\mathrm{d}}=30.6 \mu \mathrm{mol} \mathrm{L}{ }^{-1}$ and $Q_{\max }=2.92 \mu \mathrm{mol} \mathrm{g}{ }^{-1}$ for MMIMs, and $K_{\mathrm{d}}=47.5 \mu \mathrm{mol} \mathrm{L}^{-1}$ and $Q_{\max }=0.76 \mu \mathrm{mol} \mathrm{g}^{-1}$ for MNIMs, respectively, and thus the imprinting factor ( $Q_{\max }$ ratio value) was calculated as 3.84. The results suggested that the MMIMs had specific binding sites for the template molecule.

To evaluate the binding isotherms of MMIMs, four adsorption isotherm models, including Langmuir, Freundlich, Redlich-Peterson and Langmuir-Freundlich, were employed, ${ }^{24}$ as shown in Fig. 5B. Their corresponding equations and parameters for adsorption of $17 \beta-\mathrm{E} 2$ onto the three polymers are listed in Table S4. $\dagger$ Detailed descriptions for model fitting are given in the ESI. $\dagger$ It can be seen that for all the three polymers, the Langmuir-Freundlich isotherm model yielded the best fitting among the four models with correlation coefficients $\left(R^{2}\right)$ of 0.9995, representing its ability to simultaneously model both subsaturation and saturation behaviors. ${ }^{26}$ In addition, the MMIMs provided the highest concentration of binding sites per gram of polymers $\left(N_{\mathrm{t}}=212.8 \mu \mathrm{mol} \mathrm{g}{ }^{-1}\right)$ and the largest median binding affinity $\left(\alpha=5.677 \mathrm{~g} \mu \mathrm{mol}^{-1}\right.$ ), indicating an excellent imprinting effect due to the presence of a number of specific binding sites on the MMIMs.

Dynamic binding experiments were conducted to assess the mass transfer properties of the MMIMs. Fig. 6A shows the timedependent increase in the amount of $17 \beta-\mathrm{E} 2$ adsorbed by MMIMs and MMIMs-Control. As can be seen in the figure, the MMIMs demonstrated significantly higher dynamic binding performances than that of MNIMs, which indicated that the uniform spherical structure and larger specific surface area of the MMIMs were favorable to rapid mass transfer.

As for the dynamic models of MMIMs, pseudo-first-order, pseudo-second-order, Elovich and intraparticle diffusion ${ }^{27-29}$ for $17 \beta-\mathrm{E} 2$ are displayed in Fig. $6 \mathrm{~B}$, and the related equations and fitting results are listed in Table S5. $\dagger$ Detailed descriptions for the model fitting could be found in ESI. $\uparrow$ As can be seen in the figure, the pseudo-second-order model could better describe the time effect on the adsorption system than other kinetic models, which provided the most suitable correlation for the adsorption with the highest correlation coefficient of 0.9966. The pseudo-second-order equation can be expressed as follows:
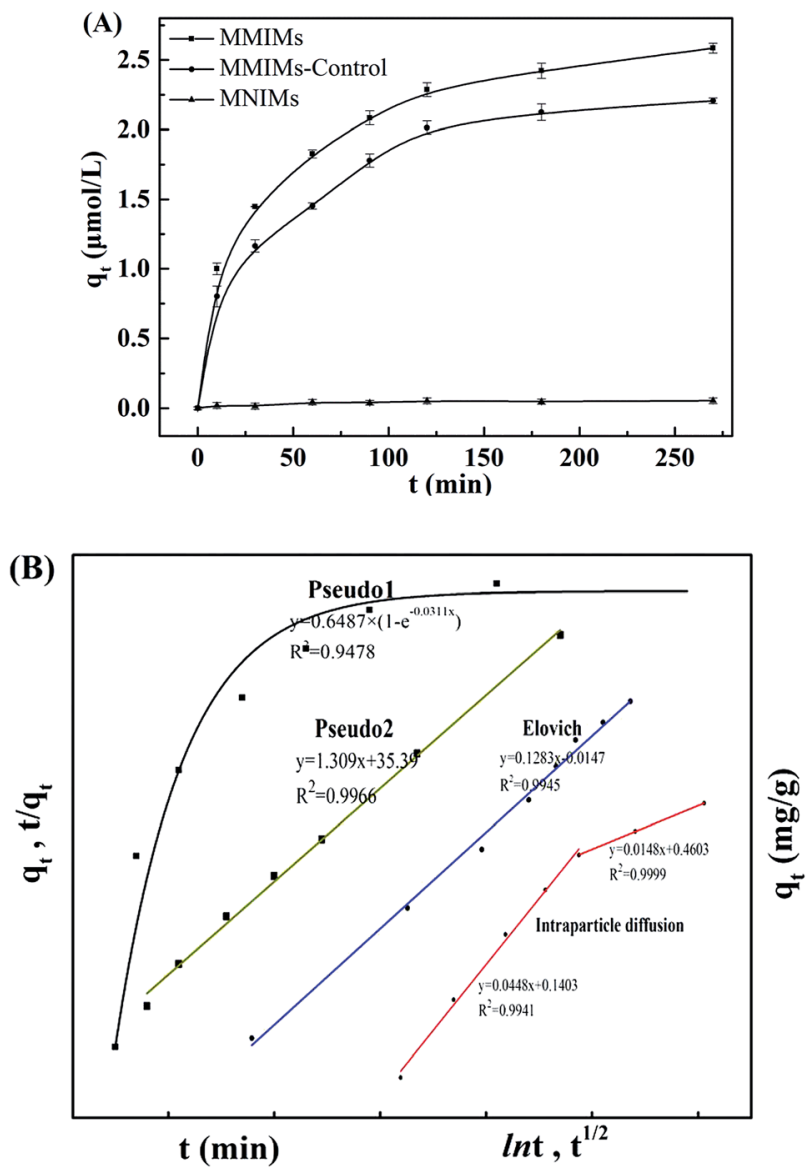

Fig. 6 (A) Kinetic binding of 17 $\beta$-E2 onto MMIMs, MMIMs-Control and MNIMs and (B) pseudo-first-order, pseudo-second-order, Elovich and intraparticle diffusion kinetic models for 17 $\beta$-E2 towards MMIMs. Experimental conditions: template concentration, $60 \mathrm{mg} \mathrm{L}^{-1} ; \mathrm{V}, 2 \mathrm{~mL}$; polymer, $20 \mathrm{mg}$; room temperature. 

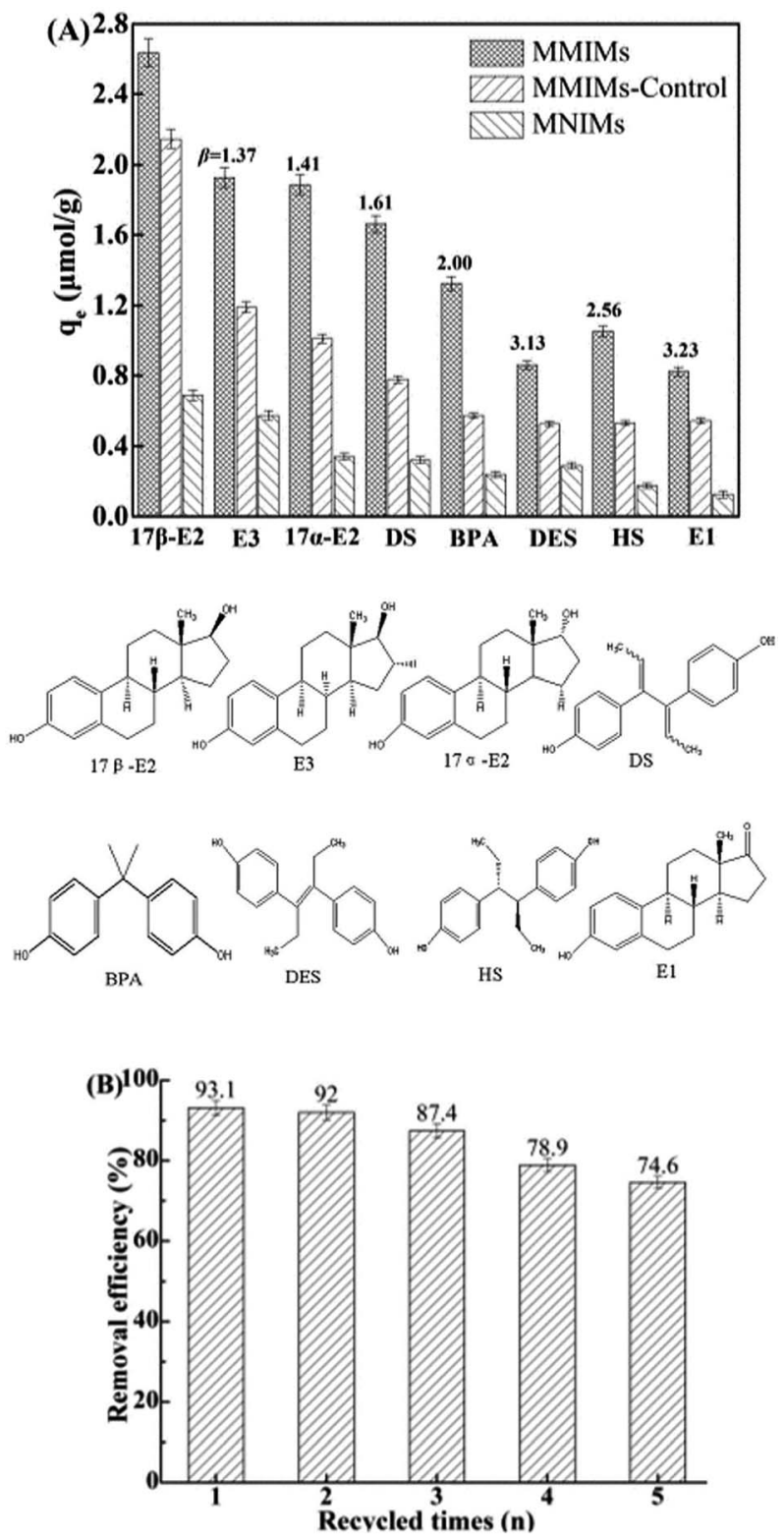

Fig. 7 (A) Binding capacities of MMIMs, MNIMs and MMIM-Control for eight PEEs (up), and their chemical structures (below). (B) Removal efficiency of MMIMS-MSPE in five recycled times (experimental conditions for (A): polymer, $20 \mathrm{mg}$; 17 $\beta$-E2 concentration, $60 \mathrm{mg} \mathrm{L}^{-1}$; $V, 2.0 \mathrm{~mL}$; adsorption time, $24 \mathrm{~h}$; room temperature. The selectivity factor was marked $\beta=q$ (template)/q(competitive molecule); and (B) polymer, $80 \mathrm{mg}$; $17 \beta-E 2,1.0 \mathrm{mg} \mathrm{L}^{-1} ; \mathrm{V}, 2.0 \mathrm{~mL}$; incubating time, $3 \mathrm{~h}$; eluting, $2 \mathrm{~mL}$ methanol-acetic acid solution $(9: 1, \mathrm{v} / \mathrm{v})$; redissolved solution, $2.0 \mathrm{~mL}$ acetonitrile).

$$
\frac{t}{q_{t}}=\frac{1}{k_{2} q_{\mathrm{e}}^{2}}+\frac{t}{q_{\mathrm{e}}}
$$

where $q_{\mathrm{t}}$ is the instantaneous adsorption amount of 17 $\beta$-E2 in the adsorbent at time $t, k_{2}$ is the adsorption rate constant, and $q_{\mathrm{e}}$ is the adsorption amount at equilibrium. The obtained $q_{\mathrm{e}}$ of $2.81 \mu \mathrm{mol} \mathrm{g}^{-1}$ calculated from the pseudo-second-order model agreed well with the $q_{\mathrm{e}}$ of $2.64 \mu \mathrm{mol} \mathrm{g}^{-1}$ from the experimental results. Thus, the adsorption could be deduced to follow the pseudo-second-order kinetics model.
In order to evaluate the competitive recognition ability of the obtained MMIMs, seven PEEs (E3, 17 $\alpha$-E2, DS, BPA, DES, HS and E1) as structural analogues were used. As seen from Fig. 7A (up), MMIMs presented a higher binding capacity for $17 \beta$-E2 than that for those competitive PEEs. The binding capacities and selectivity factors of the MMIMs for E3, $17 \alpha$-E2 and DS were close and higher than the other four PEEs, as their structures are more similar to that of $17 \beta$-E2 (Fig. 7A, below). Moreover, compared with the MMIMs-Control, the MMIMs showed obviously higher binding capacity, revealing the utilization of RAFT as an ideal strategy could greatly improve the binding capacity and mass transfer rate of the imprinted materials. However, MNIMs adsorbed much less template molecules, and there was no significant difference in binding capacity between the competitive PEEs and $17 \beta$-E2, since there were no tailor-made recognition sites formed in the MNIMs. Therefore, the obtained MMIMs could selectively recognize the delicate difference of $17 \beta-\mathrm{E} 2$ from its analogues.

\subsection{Application of MMIMs for removal of $17 \beta-E$}

The MMIMs were used as the sorbents in the SPE process, i.e., magnetic SPE was performed. To test the stability and reusability of the MMIMs-MSPE, five binding/removal cycles were conducted. Fig. 7B shows the removal efficiency of MMIMs for $1 \mathrm{mg} \mathrm{L}^{-1} 17 \beta$-E2 after 5 recycles. As observed, the five sequential removal efficiencies were attained of $93.1 \%, 92.0 \%, 87.4 \%$, $78.9 \%$ and $74.6 \%$ (from left to right), respectively. It showed that the magnetic separation under an external magnetic field could easily and rapidly be accomplished, and the MMIMs could effectively remove $17 \beta$-E2 in at least five recycles.

In order to assess the practical applicability of MMIMsMSPE, several real samples of water, soil and food were analyzed. Table 1 lists the removal recoveries of MMIMs applied in seawater, lake water, soil and yogurt samples spiked at three concentration levels of $17 \beta-\mathrm{E} 2$. Satisfactory recoveries were obtained, such as $76.7-108.3 \%$ with precision of $1.5-4.9 \%$ at $0.10 \mathrm{mg} \mathrm{L}^{-1}$. The MMIMs were demonstrated to be potentially applicable for highly efficient preconcentration and separation of $17 \beta$-E2 in real samples. Fig. S2 $\uparrow$ presents the typical HPLC-UV chromatograms. As can be seen in the figure, the matrix effects

Table 1 MMIMs-MSPE recoveries (\%) and relative standard deviations (RSD, \%) obtained from analysis of four different samples spiked with $17 \beta-2^{a}$

Spiked concentration $\left(\mathrm{mg} \mathrm{L}^{-1}\right)$

\begin{tabular}{lrlr} 
Samples & \multicolumn{1}{c}{0.1} & 1.0 & \multicolumn{1}{c}{10.0} \\
\hline Seawater & \multicolumn{1}{l}{$76.7^{b} \pm 4.9^{c}$} & $82.7 \pm 2.7$ & $74.2 \pm 3.4$ \\
Lake water & $86.4 \pm 4.7$ & $75.6 \pm 4.4$ & $71.7 \pm 2.8$ \\
Soil & $108.3 \pm 4.7$ & $93.4 \pm 2.6$ & $105.8 \pm 3.0$ \\
Yogurt & $88.9 \pm 1.5$ & $96.0 \pm 1.1$ & $100.3 \pm 6.0$
\end{tabular}

${ }^{a}$ Experimental conditions: polymer, $80 \mathrm{mg}$; sample volume, $2 \mathrm{~mL}$; incubating time, $3 \mathrm{~h}$; separation, magnet; eluting, $2 \mathrm{~mL}$ methanolacetic acid solution $(9: 1, \mathrm{v} / \mathrm{v})$; redissolved solution, $2 \mathrm{~mL}$ acetonitrile. ${ }^{b}$ Average value from three individual experiments. ${ }^{c} n=3$. 
were reduced and the spiked $17 \beta$-E2 compounds were concentrated by MMIMs-MSPE, indicating that MMIMs had excellent imprinting efficiency. Moreover, the magnetic property would enable the MMIMs-MSPE more attractive. Compared to conventional SPE, the MSPE provided a simple fast magnetic separation and enrichment procedure, preventing from the complex pretreatments such as filtration and centrifugation.

\section{Conclusions}

In summary, a convenient and cost-effective magnetic coreshell imprinted microsphere for selective recognition and effective removal of $17 \beta-\mathrm{E} 2$ in complicated matrices was prepared through a one-pot synthesis by RAFT living precipitation polymerization. The obtained MMIMs showed strong response to an external magnetic field with a rapid removal efficiency and displayed high binding capacity and selectivity towards the template. The MMIMs offered satisfactory recoveries for the template when used for MSPE and were successfully applied in the enrichment and removal of trace $17 \beta$-E2 from environmental and food samples with good stability and repeatability. This work provided an excellent platform for the removal of PEEs and demonstrated a bright future for matrices purification and pollution abatement.

\section{Acknowledgements}

This work was financially supported by the National Natural Science Foundation of China (21105117, 31160317, 21175084, 21307052 and 21275068), the Natural Science Foundation of Shandong Province of China (ZR2013BL006), and the Key Laboratory of Coastal Zone Environmental Processes, YICCAS (201207).

\section{Notes and references}

1 J. Kaiser, Science, 2000, 290, 695.

2 N. Yildirim, F. Long, C. Gao, M. He, H. Shi and A. Gu, Environ. Sci. Technol., 2012, 46, 3288.

3 P. Matthiessen and I. Johnson, Environ. Pollut., 2007, 146, 9.

4 M. Maynadier, P. Nirdé, J. Ramirez, A. Cathiard, N. Platet, M. Chambon and M. Garcia, Hormonal Carcinogenesis $V$, Springer, New York, 2008, p. 485.
5 Z. Meng, W. Chen and A. Mulchandani, Environ. Sci. Technol., 2005, 39, 8958.

6 L. Chen, S. Xu and J. Li, Chem. Soc. Rev., 2011, 40, 2922.

7 J. Ma, L. Yuan, M. Ding, S. Wang, F. Ren, J. Zhang and X. Zhou, Biosens. Bioelectron., 2011, 26, 2791.

8 Y. Shi, D. Peng, C. Shi, X. Zhang, Y. Xie and B. Lu, Food Chem., 2011, 126, 1916.

9 M. Noir, F. Plieva, T. Hey, B. Guieysse and B. Mattiasson, J. Chromatogr. A, 2007, 1154, 158.

10 G. Pan, Y. Zhang, X. Guo, C. Li and H. Zhang, Biosens. Bioelectron., 2010, 26, 976.

11 M. Titirici and B. Sellergren, Chem. Mater., 2006, 18, 1773.

12 S. Xu, J. Li and L. Chen, Talanta, 2011, 85, 282.

13 S. Xu, J. Li and L. Chen, J. Mater. Chem., 2011, 21, 4346.

14 T. Zhou, L. Jorgensen, M. Mattebjerg, I. Chronakis and L. Ye, RSC Adv., 2014, 4, 30292.

15 S. Xu, C. Guo, Y. Li, Z. Yu, C. Wei and Y. Tang, J. Hazard. Mater., 2014, 264, 34.

16 C. Alvarez lorenzo, C. Gonzalez Chomon and A. Concheiro, Smart Materials for Drug Delivery, 2013, vol. 2, p. 228.

17 G. Martins, J. Mano and N. Alves, Langmuir, 2011, 27, 8415.

18 Y. Li, X. Li, J. Chu, C. Dong, J. Qi and Y. Yuan, Environ. Pollut., 2010, 158, 2317.

19 S. Xu, J. Li, X. Song, J. Liu, H. Lu and L. Chen, Anal. Methods, 2013, 5, 124.

20 Y. Li, C. Dong, J. Chu, J. Qi and X. Li, Nanoscale, 2011, 3, 280.

21 P. Dramou, P. Zuo, H. He, L. Pham-Huy, W. Zou, D. Xiao and C. Pham-Huy, J. Chromatogr. A, 2013, 1317, 110.

22 T. Jiang, L. Zhao, B. Chu, Q. Feng, W. Yan and J. Lin, Talanta, 2009, 78, 442.

23 P. Manesiotis, L. Fitzhenry, G. Theodoridis and P. Jandera, Anal. Tech. Scientists, 2012, 4, 457.

24 R. Krupadam, B. Bhagat, S. Wate, G. Bodhe, B. Sellergren and Y. Anjaneyulu, Environ. Sci. Technol., 2009, 43, 2871.

25 E. Zoelen, R. Kramer, H. Moerkerk and J. Veerkamp, Trends Pharmacol. Sci., 1998, 19, 487.

26 W. Ma, F. Ya, M. Han and R. Wang, J. Hazard. Mater., 2007, 143, 296.

27 Y. Ho and G. McKay, Process Biochem., 1999, 34, 451.

28 J. Zhu, S. Wei, H. Gu, S. Rapole, Q. Wang, Z. Luo and Z. Guo, Environ. Sci. Technol., 2011, 46, 977.

29 X. Cai, J. Li, Z. Zhang, F. Yang, R. Dong and L. Chen, ACS Appl. Mater. Interfaces, 2014, 6, 305. 